\title{
Serum interleukin- 6 as a prognostic marker in neonatal calf diarrhea
}

\author{
Stephani Fischer, ${ }^{*}$ Rolf Bauerfeind, $†$ Claus-Peter Czerny, ${ }^{*}$ and Stephan Neumann $\ddagger^{1}$ \\ *Department of Animal Sciences, Institute of Veterinary Medicine, Division of Microbiology and Animal Hygiene, Faculty of Agricultural Sciences, \\ Georg-August University Goettingen, Goettingen 37077, Germany \\ †Institute of Hygiene and Infectious Diseases of Animals, Justus-Liebig University, Giessen 35392, Germany \\ ¥Small Animal Clinic, Institute of Veterinary Medicine, Faculty of Agricultural Sciences, Georg-August University Goettingen, Goettingen 37077 , \\ Germany
}

\begin{abstract}
Neonatal calf diarrhea is still one of the most important diseases in calf rearing, and severe diarrhea has a marked effect on animal welfare. Furthermore, significant economic losses can result from this disease due to high mortality rates, high medical costs, and low weight gain. To avoid a fatal outcome of the disease, it is crucial that vulnerable calves are identified as early as possible. Interleukin- 6 is described as an early and reliable prognostic marker in several diseases. In this study, 20 scouring calves were tested by ELISA for their IL-6 serum concentrations. Samples were collected twice, at the beginning of diarrhea and 7 to $10 \mathrm{~d}$ later. Regarding the clinical outcome after 7 to $10 \mathrm{~d}$, calves were classified as recovered or nonrecovered. A receiver operating characteristic analysis was conducted to determine the prognostic value of IL-6 for the progress of clinical symptoms. At the beginning of diarrhea, the IL-6 concentration was significantly higher in nonrecovering calves compared with those that recover 7 to $10 \mathrm{~d}$ after the onset of diarrhea. Interleukin- 6 proved to be a useful additional parameter in the clinical examination. High initial IL-6 values can support the decision for closer monitoring and an adapted therapeutic strategy for the respective calves. This may help to prevent unnecessary animal suffering and reduce economic losses. Key words: neonatal calf diarrhea, interleukin 6, ELISA, bovine rotavirus, Cryptosporidium parvum
\end{abstract}

\section{INTRODUCTION}

Neonatal calf diarrhea poses a marked threat to animal welfare and causes significant economic losses due to high mortality, higher medical costs, and low weight gain (Waltner-Toews et al., 1986; Warnick et al., 1995; Mohd Nor et al., 2012). Infectious and noninfectious

\footnotetext{
Received December 10, 2015.

Accepted April 18, 2016.

${ }^{1}$ Corresponding author: sneuman@gwdg.de
}

causes can lead to severe diarrhea in calves. In the first 4 wk of life, infections with bovine coronavirus (BCV), bovine rotavirus (BRV), Cryptosporidium parvum, and enterotoxic Escherichia coli in particular can cause inflammatory and functional damage to the intestine (Kaske, 1993; Al Mawly et al., 2015). Insufficient management of the diseased calves can quickly result in a fatal outcome (Meganck et al., 2014). A reliable prognostic marker for the progress of diarrhea would enable dairy farmers and veterinarians to monitor critical patients more closely.

In several disorders, interleukins and especially IL-6, appear to be such a marker of early inflammation and prognosis (Rincon, 2012). The helical 208-AA glycoprotein IL-6 is produced by several cell types and mainly operates as an inducer of the acute phase response (Droogmans et al., 1992; Barton, 1997). Therefore, it is upregulated in the early stage of an inflammatory process and rapidly decreases after 12 to $24 \mathrm{~h}$ (Okabe et al., 1996; Volante et al., 2004). Thus, IL-6 could serve as an early marker for inflammation because serum titers increase before the inflammation becomes clinically apparent.

Several studies in human medicine investigated IL-6 as a prognostic marker related to a specific pathogen. Children displaying hemolytic uremic syndrome due to an infection with enterohemorrhagic $E$. coli showed higher levels of IL-6 when the disease became more severe in nature (e.g., anuria, extrarenal manifestation, chronic renal sequelae; Karpman et al., 1995). Likewise, high IL-6 values were present in children suffering from shigellosis when complications arose during illness (de Silva et al., 1993). For humans and pigs, it was shown that serum IL-6 values were elevated in individuals displaying diarrhea due to an infection with rotavirus (Jiang et al., 2003; Azevedo et al., 2006).

Despite the direct connection of IL-6 to a specific pathogen, IL-6 has also been established as a reliable marker for the clinical outcome of human patients, independent from the type of underlying disease. Interleukin-6 was prognostic for sepsis and mortality 
in adult humans suffering from a bacterial infection, regardless of what pathogen was causatively involved (Moscovitz et al., 1994). This was confirmed by another study among severely infected humans at an emergency department, where nonsurvivors had higher IL-6 serum concentrations at the initial examination than survivors (Wilhelm et al., 2012). In hospitalized human neonates, an increased IL-6 serum level was proven $100 \%$ sensitive in detecting individuals who developed a clinical sepsis at later stages of their disease (Buck et al., 1994). In veterinary medicine, IL-6 was also a reliable parameter to monitor dogs in intensive care units (Schüttler and Neumann, 2015). The mean IL-6 concentration of these dogs was significantly higher in the nonsurvivor group compared with survivors.

Only a small number of studies covered the diagnostic and prognostic potential of IL-6 in cattle. For instance, IL-6 values in cows suffering from subclinical mastitis were elevated in the early stages of the disease, even before the number of somatic cells increased (Sakemi et al., 2011). Other investigators focused on the IL-6 serum values in pregnant cows prepartum (Ishikawa et al., 2004). They found high IL-6 serum concentrations in those cows that developed endometritis postpartum. In the case of coliform mastitis, serum IL-6 was shown to be significantly higher in nonsurvivors when sampled at d 0 to 9 of the onset of clinical symptoms (Nakajima et al., 1997).

As investigations of serum IL-6 concentrations in the case of neonatal calf diarrhea are lacking and the improvement of therapy would benefit from a reliable prognostic marker, this study investigated the prognostic quality of IL- 6 for the progress of clinical symptoms in neonatal calf diarrhea. Therefore, a controlled cohort study over $10 \mathrm{~d}$ was carried out on 20 scouring calves. Calves were monitored for clinical changes as well as changes in body homeostasis (e.g., hematology, clinical chemistry) related to diarrhea. For the determination of the prognostic potential, scouring calves were divided into 2 groups, regarding their classification at d 10 as recovered or nonrecovered. A receiver operating characteristic (ROC) analysis with calculation of the area under the curve (AUC) was carried out to prove the accuracy of IL-6 determination in serum for the estimation of the prognosis.

\section{MATERIALS AND METHODS}

\section{Animals}

Calves were housed on a commercial dairy farm near Goettingen, Lower Saxony, Germany. The farm is organized in terms of 3 locations. One location is for the 500 milk yielding and late pregnant cows, one for the calves and the last one for the offspring, older than 8 wk. Biotechnical measures directly after birth included disinfection of the umbilicus and application of earmarks. Four liters of colostrum was fed to the calves via tube feeder within the first hour of life. The colostrum came from the farm's colostrum bank and was controlled for sufficient quality with a colostrometer. Within $24 \mathrm{~h}$ after birth, newborn calves were brought to a special calf location, which was geographically separated from the barn for the milk-yielding cows. The newborn calves were sheltered outdoors in igloos individually and were fed pasteurized waste milk ad libitum in the first week after birth. Then, a commercial milk replacer $(50 \%$ skim milk) displaced the milk. From wk 3 on, starter grain was offered and calves had free access to water. Calves were weaned 4 wk after birth.

Sampling was conducted during January to July 2014. All Holstein Friesian calves displaying diarrhea were included in the study. Calves were excluded when showing any other diseases, such as respiratory tract infections or infections of the umbilicus. Finally, the group of scouring calves consisted of 20 animals (age $12.9 \pm 2.0 \mathrm{~d} ; 89 \%$ female). Scouring calves were sampled once at the first day of evident symptoms and again 7 to $10 \mathrm{~d}$ later. At these time points, all animals passed a complete clinical examination with information on heart rate, breathing rate, temperature, dehydration, and general condition. Diarrhea scoring was undertaken using an already established scoring system, evaluating the fecal consistency on a 4-point scale from normal (0) to totally liquid (4) (Hasoksuz et al., 2002). All calves received an oral electrolyte solution from the beginning to the end of diarrhea and were initially treated with $0.5 \mathrm{mg} / \mathrm{mL}$ of meloxicam. Throughout the study period, calves were monitored twice per day by the animal caretakers.

After the sampling period, the calves were further divided into 2 subgroups: one subgroup $(\mathrm{n}=11$; age $15.0 \pm 2.8 \mathrm{~d}$ ) included calves that clinically recovered 7 to $10 \mathrm{~d}$ after the first symptoms appeared and the other subgroup $(\mathrm{n}=9$; age $10.2 \pm 1.7 \mathrm{~d}$ ) included calves evaluated as nonrecovered, because they were still showing diarrhea, still being medically treated, or showing other abnormalities in the clinical examination (e.g., reduced general condition). All clinical examinations were performed by the same veterinarian to avoid an examiner bias.

\section{Samples}

All samples were acquired with the owner's consent and the procedures were carried out in accordance with the German Protection of Animals Act under the supervision of the Commissioner for Animal Welfare, Fac- 
ulty of Agriculture, University of Goettingen. For the purpose of laboratory analysis, $5 \mathrm{~mL}$ of EDTA-treated blood and $10 \mathrm{~mL}$ of whole blood for serum harvest were collected by jugular venipuncture. Stool samples were collected from the rectum following digital stimulation. All samples were cooled immediately after collection and brought to the laboratory within $6 \mathrm{~h}$. Blood samples and feces were processed directly. Serum was stored at $-20^{\circ} \mathrm{C}$ until further use in the IL-6 ELISA.

\section{Hematology and Clinical Chemistry}

The complete blood count and the serum clinical chemistry were analyzed in all calves to ensure the healthiness of the control group and to determine pathological changes due to diarrhea. The EDTA blood samples were used for a complete blood count using a CellDyn 3500 Analyzer (Abbott GmbH \& Co. KG, Wiesbaden, Germany). Because differentiation of bovine lymphocytes and monocytes can be difficult, the results of the CellDyn 3500 Analyzer were verified by a manual blood count. Serum samples were aliquoted to $500-\mu \mathrm{L}$ aliquots and stored at $-20^{\circ} \mathrm{C}$. One aliquot was used directly for the clinical chemistry analysis with the Konelab 20i (Thermo Fisher Scientific Inc., Dreieich, Germany).

\section{Stool Analysis}

Stool samples were analyzed by a commercial lateral immunochromatography test (Bio-X Diagnostics, Rochefort, Belgium) to identify the causative pathogen for diarrhea, such as BCV, BRV, Cryptosporidium parvum, and E. coli F5 (K99).

\section{IL-6 ELISA}

The IL-6 serum concentrations were determined using the commercial High Sensitive ELISA Kit for bovine IL-6 (USCN Life Science, Wuhan, China). First, standard and samples were added in duplicate to the pre-coated plate and incubated for $2 \mathrm{~h}$ at $37^{\circ} \mathrm{C}$. Subsequently, all liquid was removed and Detection Reagent A (biotin-conjugated antibody specific to IL-6) was added for $1 \mathrm{~h}$ at $37^{\circ} \mathrm{C}$. The plate was washed 3 times with wash solution $(1 \times)$ and Detection Reagent $\mathrm{B}$ (avidin-conjugated horseradish peroxidase) was added and incubated at $37^{\circ} \mathrm{C}$ for $30 \mathrm{~min}$. The plate was washed 5 times with wash solution before substrate solution (TMB substrate) was added for $20 \mathrm{~min}$ at $37^{\circ} \mathrm{C}$. Color development was stopped with stop solution $\left(\mathrm{H}_{2} \mathrm{SO}_{4}\right)$ and optical densities were measured in all wells at $450 \mathrm{~nm}$ in the Sunrise microplate reader
(Tecan, Maennedorf, Switzerland). For data analysis, average optical densities were calculated and blank reduction was performed. Standard curves were generated and sample concentrations were determined using the CurveExpert 1.4 software (CurveExpert, Daniel G. Hyams, Hixson, TN; https://www.curveexpert.net/). A detection limit of $1.56 \mathrm{pg}$ of IL-6/mL was reported by the kit manufacturer. The coefficient of variation for the intra-assay test precision was $<7 \%$.

\section{Statistical Analysis}

Results were shown as mean values \pm 2 standard errors. Data from the clinical examination, the hematology and the IL-6 ELISA were tested for normal distribution.

Data of recovering and nonrecovering animals were compared at both sampling time points. Regarding the IL-6 values in these groups, further statistical tests were performed. A 2-way ANOVA was calculated to investigate the interaction of group (recovering, nonrecovering) and sampling time point (first, second). To investigate a change in the odds of IL- 6 concentrations at the onset of diarrhea (first sampling time point), to predict a prolonged course of diarrhea (nonrecovering), a univariate logistic regression analysis was performed. The goodness-of-fit of the model was assessed by performing an Omnibus test of model coefficients and a Hosmer-Lemeshow test, as well as calculating the Nagelkerke $R^{2}$. The influence of the variable IL-6 was estimated using the Wald statistic.

As the age of calves is described to be a risk factor to develop diarrhea, this variable is analyzed in the same way regarding the age of calves at the first sampling time point (Pare et al., 1993; Gulliksen et al., 2009). Subsequently, a multivariate logistic regression analysis was performed, with both variables (IL-6, age) and a potential interaction effect between these variables. The goodness-of-fit for this analysis was conducted as for the univariate analysis. Using forward model selection, a $P$-value of $<0.05$ was chosen as the cut-off for the respective variable to stay in the model. To further evaluate the prognostic quality of IL-6, a ROC curve was generated with the IL-6 values of the recovering and the nonrecovering animals at the first sampling time point. The sensitivity was defined as the percentage of samples which were classified correctly as nonrecovering. The specificity was defined as the percentage of samples which were classified correctly as recovering. The AUC was calculated by trapezoidal summation, and its statistical significance was estimated. All analyses were carried out using STATISTICA 12 software [StatSoft (Europe) GmbH, Hamburg, Germany] and 
IBM SPSS Statistics 20.0 (IBM Corp., 2011, IBM SPSS Statistics for Windows, Armonk, NY). The statistical significance level was set at $P<0.05$.

\section{RESULTS}

\section{Clinical, Biochemical, and Hematological Findings}

The results of the clinical and laboratory examination are shown in Table 1. Most of the recovering calves showed mild symptoms of diarrhea (score $2, \mathrm{n}=9$ ), and none of them displayed diarrhea at the second sampling time point. Severe diarrhea was only seen in the nonrecovering group (score $4, \mathrm{n}=2$ ), in which 5 calves were still displaying diarrhea 7 to $10 \mathrm{~d}$ after the onset of symptoms. The clinical chemistry and the hematology revealed no significant differences between recovering and nonrecovering animals at the first or second sampling time point (data not shown). Despite the number of white blood cells at the first sampling being within the reference values $\left(5-10 \times 10^{9}\right.$ cells/L) for recovering $\left(\mathbf{R G} ; 6.5 \pm 1.3 \times 10^{9}\right.$ cells $\left./ \mathrm{L}\right)$ and nonrecovering (NRG; $10.3 \pm 2.1 \times 10^{9}$ cells/L) calves, animals from the NRG showed higher values than the RG animals $(P<0.01)$. This was caused by a higher number of neutrophils (RG: $2.6 \pm 0.6 \times 10^{9}$ cells/L, NRG: $6.1 \pm$ $2.4 \times 10^{9}$ cells $/ \mathrm{L}$, reference range: $1-3.5 \times 10^{9}$ cells/L) At the second sampling time point, no significant differences were detected.

\section{Detection of Diarrheagenic Pathogens in Fecal Samples}

Bovine rotavirus and Cryptosporidium parvum were identified as the diarrhea-causing pathogens in scouring calves (Table 1). Mono-infections with only 1 pathogen, as well as double infections, were detected. Pathogens changed from the first to the second sampling time points, in both the RG and the NRG. In the RG infections with BRV decreased from 3 to 1 positive samples, infections with C. parvum decreased from 5 to 4 positive samples and double infections were no longer detected at the second sampling. In the NRG infections with $C$. parvum decreased from 7 to 4 positive samples, double infections decreased from 2 to 1 and 2 new BRV infections were detected at the second sampling time point.

\section{Interleukin-6 in Serum Samples}

Interleukin- 6 results are presented as means $\pm 2 \mathrm{SE}$ (Figure 1). No significant interaction was found between the effects of group (RG, NRG) and sampling time point $(1,2)$ on serum IL-6 $[F=(1,36)=1.776$, $P=0.191]$.
In terms of disease progression, initially the NRG calves showed a higher $(P=0.02)$ IL-6 mean value $(42.3 \pm 6 \mathrm{pg} / \mathrm{mL})$ than the RG calves $(31.8 \pm 5.5 \mathrm{pg} /$ $\mathrm{mL})$.

A logistic regression was conducted to determine the effects of age and IL-6 at the onset of diarrhea (first sampling time point) on the odds for calves to develop a prolonged course of diarrhea (Table 2). The univariate logistic regression model for IL-6 (model 1) was statistically significant, as the $P$-value for the chi-squared statistic (Omnibus test of model coefficients) was 0.014 and the Hosmer-Lemeshow test showed no statistical significance $(P=0.640)$. The model explained $34 \%$ (Nagelkerke $\mathrm{R}^{2}$ ) of variance. The Wald statistic revealed a significant influence of IL-6 in the model $(P=0.041)$. The odds for calves developing a prolonged course of the disease increase by $14 \%$ for each unit of IL- 6 (odds ratio $1.14 ; 95 \%$ CI 1.0-1.3). A $t$-test revealed the age of the RG and the NRG animals being significantly different $(P=0.01)$. A univariate logistic regression model was conducted to test for a significant influence of the variable "age" on the odds for a prolonged course of diarrhea. As the analysis revealed significance $(P=$ 0.039 ), a multivariate logistic regression was performed including IL-6 and age (model 2). In this model, none of the variables showed significance. Additionally, no confounding by age was noted as there was less than a $20 \%$ change in the coefficient for IL- 6 with the addition of age in the model. Furthermore, even the interaction term (age $\times$ IL-6; model 3 ) showed no significance when included in the model.

The value of IL-6 as a prognostic marker for the clinical outcome of scouring calves was further evaluated in a ROC analysis (Figure 2). The ROC analysis for IL-6 values of the diarrhea group at the beginning of diarrhea revealed a cut-off of $38.7 \mathrm{pg} / \mathrm{mL}$, with a sensitivity of $77 \%$ and specificity of $82 \%$ for identifying calves with a prolonged recovery period. The area under the curve $(\mathrm{AUC}=0.808,95 \%$ CI: $0.609-1, P=0.02)$ showed a good predictive ability for IL-6 to discriminate between RG and NRG animals at the onset of diarrhea.

\section{DISCUSSION}

In this study, the prognostic value of the IL-6 serum concentration was investigated over the course of neonatal calf diarrhea. The main result was that calves developing a prolonged course of the disease showed significantly higher IL-6 values at the onset of symptoms.

Initially, hematology and clinical chemistry were analyzed. The calves showed elevated levels of neutrophils, whereas the total number of white blood cells was within the reference range. The neutrophilia was re- 


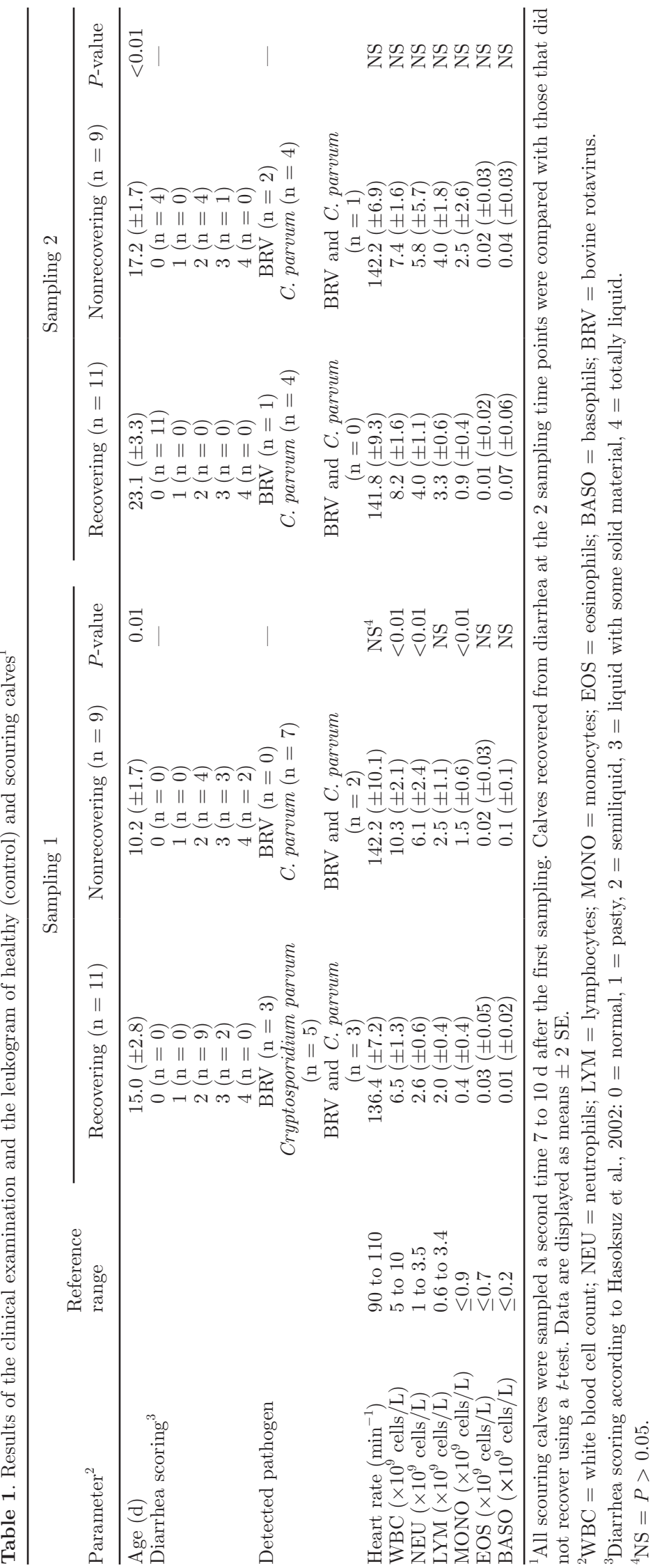




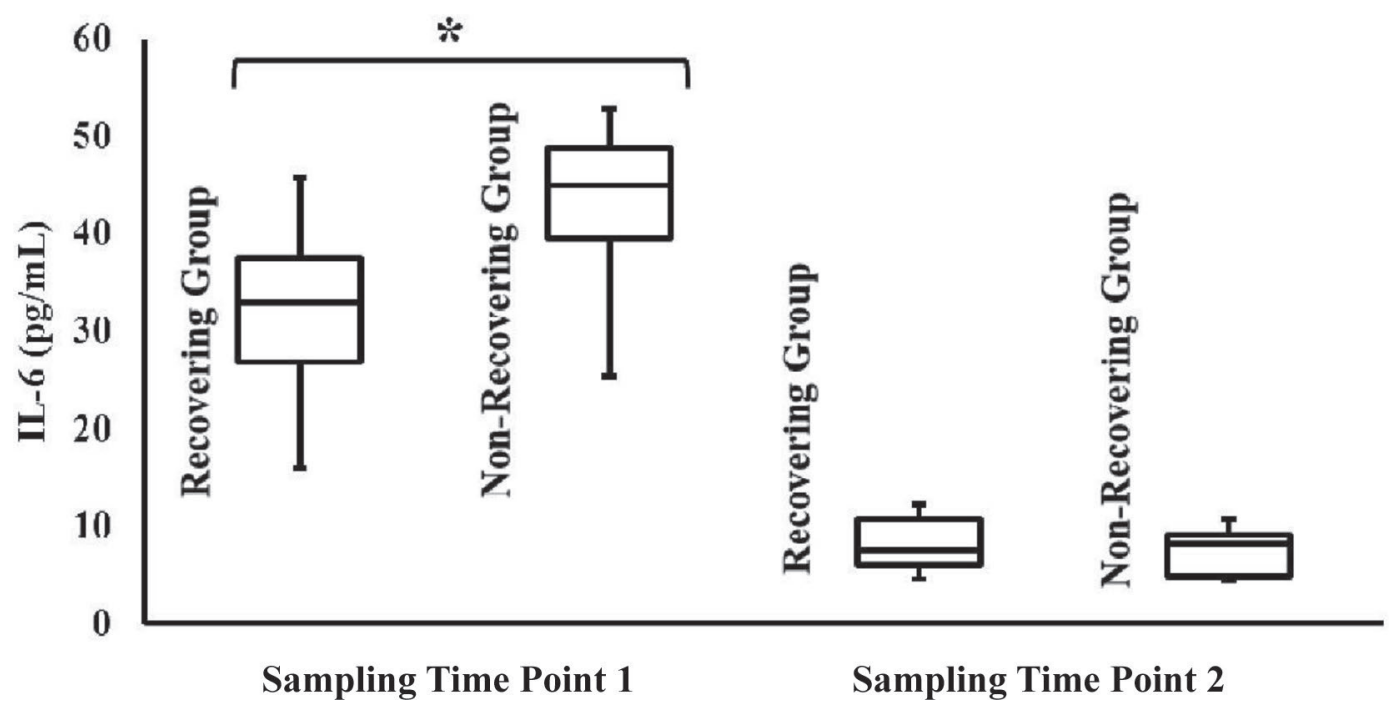

Figure 1. Comparison of IL-6 in scouring Holstein Friesian calves with different clinical outcome. The scouring calves were grouped by clinical recovery 7 to $10 \mathrm{~d}$ after (second sampling time point) the onset of diarrhea (first sampling time point). The IL-6 serum concentrations of the recovering group $(\mathrm{n}=11)$ and the nonrecovering group $(\mathrm{n}=9)$ were compared on both sampling time points. ${ }^{*} P<0.05$. Recovering group: calves were clinically recovered 7 to $10 \mathrm{~d}$ after the onset of diarrhea. Nonrecovering group: calves were not clinically recovered 7 to $10 \mathrm{~d}$ after the onset of diarrhea. Upper whisker $=$ maximum, lower whisker $=$ minimum, upper box line $=$ 3rd quartile, middle box line $=$ median, lower box line $=1$ st quartile.

lated to handling stress during sampling rather than to diarrhea, as it was similar in both groups. An increase of neutrophils due to stress was already described for cattle in cases of shipping and heat stress (Kegley et al., 1997; Mitlohner et al., 2002). The differential blood count, measured by the CellDyn 3500 Analyzer, revealed increased numbers of monocytes when compared with the reference values. However, manual counting of blood smears could not confirm the monocytosis. The CellDyn 3500 Analyzer classifies cells by measuring

Table 2. Results of the logistic regression analysis for model $1^{1}$

\begin{tabular}{|c|c|c|}
\hline Item & & Model 1 \\
\hline Variable & & $\begin{array}{c}\text { IL-6 } \\
(\mathrm{n}=20)\end{array}$ \\
\hline$B^{2}$ & & 0.134 \\
\hline $\mathrm{SE}$ & & 0.066 \\
\hline$P$-value* & & 0.041 \\
\hline $\operatorname{Exp}^{3}(\mathrm{~B})$ & & 1.143 \\
\hline \multirow[t]{2}{*}{$95 \%$ CI for $\operatorname{Exp}(\mathrm{B})$} & Lower & 1.005 \\
\hline & Upper & 1.300 \\
\hline Omnibus test* & & $P=0.014$ \\
\hline Nagelkerke $\mathrm{R}^{2}$ & & 0.346 \\
\hline Hosmer-Lemeshow test* & & $P=0.640$ \\
\hline
\end{tabular}

${ }^{1} \mathrm{IL}-6$ values at the onset of diarrhea (first sampling time point) were evaluated for their odds to predict a prolonged course of diarrhea (nonrecovering).

${ }^{2}$ Regression coefficient B.

${ }^{3}$ Exponentiation of the $\mathrm{B}$ coefficient.

${ }^{*} P$-value $<0.05$ was regarded as significant. volume and granularity. It is likely that the number of monocytes was overestimated by mistaking them for large lymphocytes (Stoeber and Heubner, 1967).

The detection of diarrhea-causing pathogens revealed differences between the first and the second sampling time point in both groups (recovering, nonrecovering). In the recovering group, 5 calves still shed either BRV or C. parvum at the second sampling time point, although none of them showed diarrhea. This was described in other studies also, were BRV shedding occurred up to $11 \mathrm{~d}$ after infection, whereas diarrhea only lasts $7 \mathrm{~d}$ (Vega et al., 2015). Similarly, C. parvum detection in feces was shown to be not always connected to diarrhea in the corresponding animals (Bjorkman et al., 2015). In the nonrecovering group, 2 animals showed a BRV infection at the second sampling time point, which had not been apparent at the first sampling time point. It was suggested that this was due to a secondary infection, which is frequently described in neonatal calf diarrhea (Bartels et al., 2010; Silverlas et al., 2010). In the classification of calves as recovered or nonrecovered, the pathogen detection was less important than the results of the clinical examination when focusing on the establishment of a prognostic marker.

Serum IL-6 concentrations were greater in the diarrhea group than in controls. This is consistent with findings of an increased IL-6 gene expression in peripheral blood mononuclear cells and in intestinal epithelial cells from scouring calves ex vivo due to an infection with BRV (Aich et al., 2007; Qadis et al., 2014). 
Calves further investigated regarding the course of diarrhea and the ability of IL-6 to predict the clinical outcome after 7 to $10 \mathrm{~d}$. Therefore, they were divided into recovering and nonrecovering groups after collection of data. At the onset of diarrhea, IL- 6 was significantly greater in recovering and nonrecovering animals. Because calf age differed between the 2 groups, age was evaluated additionally. In a multivariate logistic regression model none of the variables IL-6 and age and showed significance. A possible interaction between these 2 variables was not observed. However, the univariate logistic regression model for IL-6 showed a significance influence of this variable in predicting a prolonged course of diarrhea and was therefore regarded as the most reliable model in this investigation. The odds to develop a prolonged course of diarrhea increase by $14 \%$ for each unit of IL- 6 and model could explain $34 \%$ of the variance. In literature, several other factors are discussed that increase the risk for diarrhea (Pare et al., 1993; Wiest and Klee, 1998; Bendali et al., 1999). Birth weight and calf housing (e.g., open barn vs. closed barn) were identified as potential risk factors (Pare et al., 1993; Al Mawly et al., 2015), whereas IgG serum concentrations seemed to have no effect (Pare et

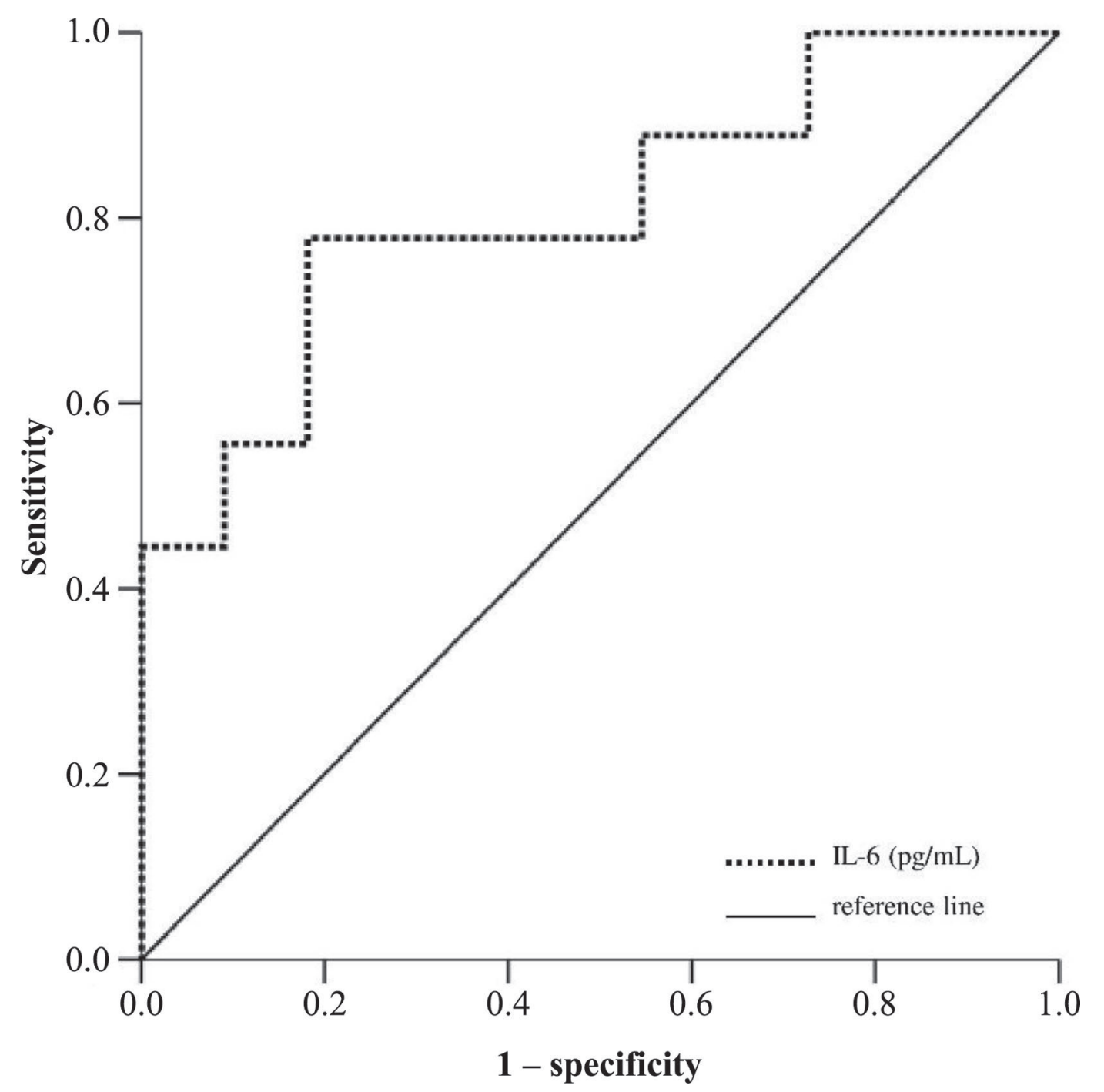

Figure 2. Receiver operating curve analysis for the evaluation of the prognostic performance of IL-6 in scouring Holstein Friesian calves. Scouring calves $(n=20)$ were grouped by their clinical recovery status 7 to $10 \mathrm{~d}$ after the onset of diarrhea (recovering group, $\mathrm{n}=11 ; \mathrm{nonrecov}-$ ering group, $\mathrm{n}=9$ ). The IL-6 serum concentrations at the onset of diarrhea were evaluated for reliability in identifying calves with a prolonged recovery period. The area under the curve (AUC) was 0.808 with a standard error under the nonparametric assumption of 0.120 , an asymptomatic significance (null hypothesis true area $=0.5$ ) of 0.02 , a lower bound of the asymptomatic $95 \%$ confidence interval at 0.609 , and an upper bound of the asymptomatic $95 \%$ confidence interval at 1.0. Recovering group: animals that were clinically recovered 7 to $10 \mathrm{~d}$ after the onset of diarrhea. Nonrecovering group: animals that did not clinically recover 7 to $10 \mathrm{~d}$ after the onset of diarrhea. 
al., 1993; Meganck et al., 2015). These parameters were not assessed in the study presented here, but could have been different in recovering and nonrecovering calves. Therefore, these parameters should be taken into consideration in future investigations, especially focusing on potential interactions between these parameters and IL-6.

The value of IL- 6 as an initial parameter for the prognosis of scouring calves was further evaluated in a ROC analysis. The AUC was used to measure the accuracy of IL-6 in distinguishing recovering from nonrecovering animals. A value of 1 was regarded as a perfect test, whereas a value of 0.5 corresponds to random chance. The AUC value of 0.808 confirmed IL- 6 as a good test for the estimation of the prognosis. Furthermore, the ROC analysis allowed the definition of a cut-off value. For the establishment of IL-6 as a prognostic marker, the sensitivity was given priority over the specificity, as a high number of false positive classifications are less important than a high number of false negative classifications. In practice, this means that it is better to monitor a calf more closely that is misclassified as nonrecovering than to overlook a calf that is prone to develop a prolonged course of diarrhea. The cut-off was set at $38.7 \mathrm{pg} / \mathrm{mL}$, with a sensitivity of $77 \%$ and specificity $82 \%$, as this was the concentration with the most appropriate balance between sensitivity and specificity.

Taken together, the results of the ROC analysis and the univariate logistic regression, the determination of IL-6 at the onset of diarrhea was a useful tool to identify calves developing a prolonged course of the disease. It is recommended that scouring calves displaying IL-6 values above $38.7 \mathrm{pg} / \mathrm{mL}$ should be given special attention, as they were prone to develop a prolonged course of diarrhea. These results underline the prognostic potential of IL-6, which was already shown for adult cattle in cases of postpartal endometritis and coliform mastitis (Nakajima et al., 1997; Ishikawa et al., 2004). Therefore, IL-6 seems highly valuable to forecast complications and possibly life-threatening progression of an infectious disease in cattle.

In conclusion, this study showed IL-6 to be a useful supplemental parameter for the estimation of disease progression in neonatal calf diarrhea. Elevated IL-6 values may lead the veterinarian to the decision to monitor scouring calves more closely and to adapt the therapeutic strategy accordingly. Thereby animal suffering and economic losses could be avoided. As the animals in our study only showed infections with BRV or Cryptosporidium parvum, further investigations are needed into all relevant pathogens for neonatal calf diarrhea [e.g., BCV, enterotoxic E. coli encoding F5 (K99) fimbriae].

\section{ACKNOWLEDGMENTS}

This study was carried out as part of the Animal Welfare in Intensive Livestock Production Systems doctoral program. The authors express thanks to the Ministry for Science and Culture of Lower Saxony (Hannover, Germany) for the financial support. We are grateful to Lynne Riddles (Goettingen, Germany) for completing the critical review of the manuscript. Another thanks goes to the Plesse Milch GmbH (Goettingen, Germany) for providing the animals included in this study and giving assistance during sampling. The authors do not have a personal or financial relationship with other people or organizations that could inappropriately influence or bias this paper. All authors have approved the paper for release and are in agreement with its content.

\section{REFERENCES}

Aich, P., H. L. Wilson, R. S. Kaushik, A. A. Potter, L. A. Babiuk, and P. Griebel. 2007. Comparative analysis of innate immune responses following infection of newborn calves with bovine rotavirus and bovine coronavirus. J. Gen. Virol. 88:2749-2761.

Al Mawly, J., A. Grinberg, D. Prattley, J. Moffat, and N. French. 2015. Prevalence of endemic enteropathogens of calves in New Zealand dairy farms. N. Z. Vet. J. 63:147-152.

Azevedo, M. S., L. Yuan, S. Pouly, A. M. Gonzales, K. I. Jeong, T. V. Nguyen, and L. J. Saif. 2006. Cytokine responses in gnotobiotic pigs after infection with virulent or attenuated human rotavirus. J. Virol. 80:372-382.

Bartels, C. J., M. Holzhauer, R. Jorritsma, W. A. Swart, and T. J. Lam. 2010. Prevalence, prediction and risk factors of enteropathogens in normal and non-normal faeces of young Dutch dairy calves. Prev. Vet. Med. 93:162-169.

Barton, B. E. 1997. IL-6: Insights into novel biological activities. Clin. Immunol. Immunopathol. 85:16-20.

Bendali, F., M. Sanaa, H. Bichet, and F. Schelcher. 1999. Risk factors associated with diarrhoea in newborn calves. Vet. Res. 30:509-522.

Bjorkman, C., L. Lindstrom, C. Oweson, H. Ahola, K. Troell, and C. Axen. 2015. Cryptosporidium infections in suckler herd beef calves. Parasitology 142:1108-1114.

Buck, C., J. Bundschu, H. Gallati, P. Bartmann, and F. Pohlandt. 1994. Interleukin-6: A sensitive parameter for the early diagnosis of neonatal bacterial infection. Pediatrics 93:54-58.

de Silva, D. G., L. N. Mendis, N. Sheron, G. J. Alexander, D. C. Candy, H. Chart, and B. Rowe. 1993. Concentrations of interleukin 6 and tumour necrosis factor in serum and stools of children with Shigella dysenteriae 1 infection. Gut 34:194-198.

Droogmans, L., I. Cludts, Y. Cleuter, R. Kettmann, and A. Burny. 1992. Nucleotide sequence of bovine interleukin-6 cDNA. DNA Seq. 2:411-413.

Gulliksen, S. M., K. I. Lie, T. Loken, and O. Osteras. 2009. Calf mortality in Norwegian dairy herds. J. Dairy Sci. 92:2782-2795.

Hasoksuz, M., A. E. Hoet, S. C. Loerch, T. E. Wittum, P. R. Nielsen, and L. J. Saif. 2002. Detection of respiratory and enteric shedding of bovine coronaviruses in cattle in an Ohio feedlot. J. Vet. Diagn. Invest. 14:308-313.

Ishikawa, Y., K. Nakada, K. Hagiwara, R. Kirisawa, H. Iwai, M. Moriyoshi, and Y. Sawamukai. 2004. Changes in interleukin-6 concentration in peripheral blood of pre- and post-partum dairy cattle and its relationship to postpartum reproductive diseases. J. Vet. Med. Sci. 66:1403-1408.

Jiang, B., L. Snipes-Magaldi, P. Dennehy, H. Keyserling, R. C. Holman, J. Bresee, J. Gentsch, and R. I. Glass. 2003. Cytokines as me- 
diators for or effectors against rotavirus disease in children. Clin. Diagn. Lab. Immunol. 10:995-1001.

Karpman, D., A. Andreasson, H. Thysell, B. S. Kaplan, and C. Svanborg. 1995. Cytokines in childhood hemolytic uremic syndrome and thrombotic thrombocytopenic purpura. Pediatr. Nephrol. 9:694-699.

Kaske, M. 1993. Physiological function of the gastrointestinal tract and pathophysiological changes in neonatal diarrhea of calves. Dtsch. Tierarztl. Wochenschr. 100:434-439. (In German)

Kegley, E. B., J. W. Spears, and T. T. Brown Jr.. 1997. Effect of shipping and chromium supplementation on performance, immune response, and disease resistance of steers. J. Anim. Sci. 75:19561964.

Meganck, V., G. Hoflack, and G. Opsomer. 2014. Advances in prevention and therapy of neonatal dairy calf diarrhoea: A systematical review with emphasis on colostrum management and fluid therapy. Acta Vet. Scand. 56:75.

Meganck, V., G. Hoflack, S. Piepers, and G. Opsomer. 2015. Evaluation of a protocol to reduce the incidence of neonatal calf diarrhoea on dairy herds. Prev. Vet. Med. 118:64-70.

Mitlohner, F. M., M. L. Galyean, and J. J. McGlone. 2002. Shade effects on performance, carcass traits, physiology, and behavior of heat-stressed feedlot heifers. J. Anim. Sci. 80:2043-2050.

Mohd Nor, N., W. Steeneveld, M. C. Mourits, and H. Hogeveen. 2012. Estimating the costs of rearing young dairy cattle in the Netherlands using a simulation model that accounts for uncertainty related to diseases. Prev. Vet. Med. 106:214-224.

Moscovitz, H., F. Shofer, H. Mignott, A. Behrman, and L. Kilpatrick. 1994. Plasma cytokine determinations in emergency department patients as a predictor of bacteremia and infectious disease severity. Crit. Care Med. 22:1102-1107.

Nakajima, Y., O. Mikami, M. Yoshioka, Y. Motoi, T. Ito, Y. Ishikawa, M. Fuse, K. Nakano, and K. Yasukawa. 1997. Elevated levels of tumor necrosis factor-alpha (TNF-alpha) and interleukin-6 (IL-6) activities in the sera and milk of cows with naturally occurring coliform mastitis. Res. Vet. Sci. 62:297-298.

Okabe, J., S. Tajima, O. Yamato, M. Inaba, S. Hagiwara, and Y. Maede. 1996. Hemoglobin types, erythrocyte membrane skeleton and plasma iron concentration in calves with poikilocytosis. J. Vet. Med. Sci. 58:629-634.

Pare, J., M. C. Thurmond, I. A. Gardner, and J. P. Picanso. 1993. Effect of birthweight, total protein, serum igg and packed cell volume on risk of neonatal diarrhea in calves on two California dairies. Can. J. Vet. Res. 57:241-246.

Qadis, A. Q., S. Goya, M. Yatsu, A. Kimura, T. Ichijo, and S. Sato. 2014. Immune-stimulatory effects of a bacteria-based probiotic on peripheral leukocyte subpopulations and cytokine mRNA expression levels in scouring Holstein calves. J. Vet. Med. Sci. 76:677-684.

Rincon, M. 2012. Interleukin-6: From an inflammatory marker to a target for inflammatory diseases. Trends Immunol. 33:571-577.

Sakemi, Y., Y. Tamura, and K. Hagiwara. 2011. Interleukin-6 in quarter milk as a further prediction marker for bovine subclinical mastitis. J. Dairy Res. 78:118-121.

Schüttler, J., and S. Neumann. 2015. Interleukin-6 as a prognostic marker in dogs in an intensive care unit. Vet. Clin. Pathol. 44:223228

Silverlas, C., K. de Verdier, U. Emanuelson, J. G. Mattsson, and C. Bjorkman. 2010. Cryptosporidium infection in herds with and without calf diarrhoeal problems. Parasitol. Res. 107:1435-1444.

Stoeber, M., and D. Heubner. 1967. Differentiation between monocytes and lymphocytes in smears of bovine (morphology, naphtholAS-esterase. Zentralbl. Veterinarmed. A 14:554-559. (In German).

Vega, C., M. Bok, L. Saif, F. Fernandez, and V. Parreno. 2015. Egg yolk IgY antibodies: A therapeutic intervention against group A rotavirus in calves. Res. Vet. Sci. 103:1-10.

Volante, E., S. Moretti, F. Pisani, and G. Bevilacqua. 2004. Early diagnosis of bacterial infection in the neonate. J. Matern. Fetal Neonatal Med. 16(Suppl. 2):13-16.

Waltner-Toews, D., S. W. Martin, and A. H. Meek. 1986. The effect of early calfhood health status on survivorship and age at first calving. Can. J. Vet. Res. 50:314-317.

Warnick, L. D., H. N. Erb, and M. E. White. 1995. Lack of association between calf morbidity and subsequent first lactation milk production in 25 New York Holstein herds. J. Dairy Sci. 78:2819-2830.

Wiest, D., and W. Klee. 1998. Retrospective evaluation of urea and creatinine blood levels in calves with diarrhea. Dtsch. Tierarztl. Wochenschr. 105:335-339. (In German)

Wilhelm, J., S. Hettwer, D. Hammer, M. Schurmann, A. Christoph, M. Amoury, T. Kloss, R. Finke, H. Ebelt, and K. Werdan. 2012. Outcome prediction using clinical scores and biomarkers in patients with presumed severe infection in the emergency department. Med. Klin. Intensivmed. Notfmed. 107:558-563 